\title{
Apparent movement in phenomenal space
}

\author{
GIOVANNI F. MISCEO and THADDEUS M. COWAN \\ Kansas State University, Manhattan, Kansas
}

\begin{abstract}
The spatial and temporal variables in Korte's third law of apparent movement were studied in pictorial arrays in which size constancy could be expected to prevail. The thresholds for apparent movement were determined under conditions in which two squares appeared on a plane either with or without perspective information for depth. The results suggest that apparent movement varies with the perceived depth separation only if the size of the stimulus pair is congruent with contextual depth representations. The obtained psychophysical function relating thresholds for third-dimensional movement to pictorial depth scale supports the view that apparent movement preserves gradient-of-texture information.
\end{abstract}

A well-known phenomenal effect of intermittent stimulation is apparent, or stroboscopic, movement. Apparent movement is known to depend on the rate of successive exposure to two stationary stimuli in the frontal plane and on the distance separating the two stimuli. Although both must increase together, the optimal exposure rate (threshold) depends on the separation, a relation formally referred to as Korte's third law (Koffka, 1935). The separation to which Korte's law refers, however, is ambiguous: the reference could be either to retinal (proximal) or to perceived separation. Corbin (1942) attempted to resolve this ambiguity by increasing the slant of the stimulus plane away from the observer, which decreased retinal separation and kept objective (distal) separation constant. Variations of slant had slight effect on the threshold required for apparent movement, suggesting that retinal separation in itself is not the effective factor.

From Corbin's results, however, the conclusion that perceived separation governs optimal exposure rate may be unwarranted, because peripheral factors (e.g., retinal size) varied as a function of slant and were not controlled. In an effort to resolve this problem, Attneave and Block (1973) held proximal separation constant but varied perceived separation with contextual two-dimensional depth cues for slant. The threshold for apparent movement was found to be greater with than without perspective slant information. Consequently, Attneave and Block suggested that their results, together with Corbin's, supported the view that "an approximately isotropic tridimensional model of physical space is constructed internally, and that apparent movement is based on events within this analog model" (1973, p. 301, italics ours; see also Attneave, 1972).

We wish to thank John Uhlarik, Leon Rappoport, and Richard Pringle for helpful comments and suggestions. Requests for reprints should be sent to G. F. Misceo, Department of Psychology, Kansas State University, Manhattan, Kansas 66506.
Because this constancy-of-extent (i.e., isotropic) view is important for a theory of spatial representation, we were led to put Korte's law to further tests. The view would be supported if it could be shown that distal metric information is preserved in apparent movement, a demonstration incompletely addressed in the studies reviewed. For example, Corbin's results showed that, although frontal separation increased additively, the thresholds for apparent movement did not, suggesting that the slope of the function is not constant and therefore indicating less than perfect constancy. Attneave and Block, while not systematically varying separation, found that the threshold with perspective, although greater than without, fell short of the expected threshold for depicted slant separation, again suggesting less than perfect constancy.

We extended Attneave and Block's experiments by varying perceived separation over a range of values in a textured pictorial array. This represents an advance over the single values tested by Attneave and Block and was necessary for determining the functional relation between perceived separation and optimal exposure rate. Our interest, however, was not simply in the absolute separation between context conditions, but rather in separation as defined by texture-gradient size; we sought to demonstrate that it is not the former, but, instead, the relative distal scale of pictorial depth that matters in Korte's law. It is known that relatively accurate perceptual constancies may be maintained in pictorial arrays in the sense that observers are responsive to the perceived distal rather than to the proximal properties (Smith \& Gruber, 1958; Smith, Smith, \& Hubbard, 1958; Uhlarik, Pringle, Jordan, \& Misceo, 1980). Hence, if texture-gradient size influenced apparent movement in a context in which size constancy would prevail, then the threshold should increase linearly, not with picture-plane separation, but with scale values of separation that preserve distal metric information. 


\section{METHOD}

\section{Observers}

The observers were 38 students fulfilling a requirement for an introductory psychology course. Five observers were rejected because of excessive perseverance in judgments of movement or no movement. Another three were rejected because they reported no apparent movement. Preliminary screening and training sessions never included any of the critical experimental conditions.

\section{Experimental Conditions}

Figure 1 shows a perspective representation of a square of fixed distal size. The bottom square ( $0.4 \mathrm{deg}$ in visual angle) was the standard. A comparison was selected from one of three different sets. The first set, shown in Figure 1, decreased in size according to the linear perspective defined by the foreshortened context. From the largest to the smallest comparison size, the visual angles were $0.28,0.20,0.16,0.12$, and $0.09 \mathrm{deg}$, and their respective separations (measured from the standard's to the comparison's bottom edge) in the picture plane were 29.5, $45.0,55.0,61.5$, and $66.0 \mathrm{~mm}$. In the second comparison set, although the picture-plane separations were unchanged, the perspective size change of Figure 1 was reversed by a 180-deg pictureplane rotation. Hence, the standard appeared at the top of the field, thus inverting the gradient-size correlation. In the third set, the comparison was identical in size to the standard at each of the given separations.

The standard and the comparison sets (each with five comparison sizes) were presented in two different contexts, a background that either did or did not contain perspective information for depth. In both cases, the context consisted of 20 lines. In the perspective condition, the distance between the lines varied hyperbolically to create a foreshortened depth gradient, and the thickness of the lines decreased every four lines with increasing height on the picture plane. In the no-perspective condition, the distance between the lines and the thickness of each line were constant. Thus, the overall design was a 2 (type of context) $\times 3$ (comparison set) factorial (five subjects per cell) with repeated measures on five levels of separation.

The context variation for identical size pairs was intended as a replication of Attneave and Block's conditions, with the modification of varying separation. While the expectation was that apparent movement would vary with perceived separation, it was not at all obvious that this result should be independent of size. If it were, however, then neither of the three size conditions should show differences, if perceived separation is the determinant par excellence of apparent movement. On the other hand, if size contributes something to apparent movement, then the optimal influence of perceived separation should be manifested by the gradient-size correlated condition.

\section{Procedure}

The stimuli were presented in a three-field tachistoscope (Iconix Model 6191). The standard square occurred in Stimulus Field 1,

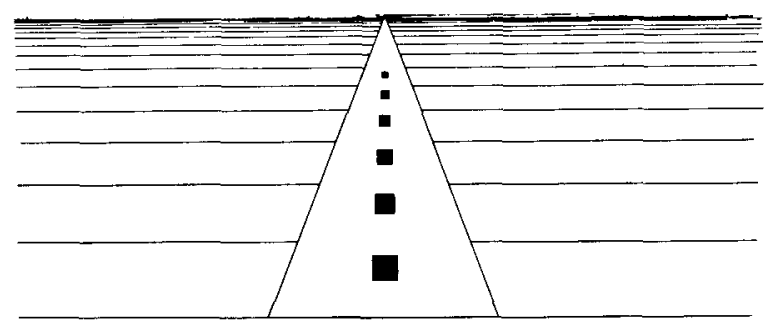

Figure 1. A two-dimensional perspective representation of the same distal-sized square in six different pictorial depth planes. a comparison square in Stimulus Field 2, and the textured background in Stimulus Field 3. The squares were continuously alternated, but the background was always visible. This procedure was designed to determine the "simultaneity threshold," that field duration at which the appearance of movement passes into the appearance of simultaneity. This measure of apparent movement was chosen because it is less variable than other measures (cf. Corbin, 1942; Orlansky, 1940; Wertheimer, 1961).

The termination of one stimulus field always coincided with the onset of the other. The two stimulus fields always had the same duration, which could be varied by the experimenter from 50 to $450 \mathrm{msec}$ in 10 -msec steps. ${ }^{1}$ In threshold determinations by the method of limits, ascending and descending series were alternated. In all series, the beginning duration was randomly chosen from a range of values determined by preliminary testing and terminated with the first reversal of judgment. The subject was instructed to report "Yes" if she/he perceived one object in apparent motion and "No" if she/he perceived two objects simultaneously flickering.

Each observer was tested individually. The stimulus field was viewed binocularly with room lights off. All participants were encouraged to maintain a head position near or touching the viewing scope of the tachistoscope. There was no fixation point because (1) in preliminary pilot work, observers reported less local adaptation without a fixation point, and (2) previous research has demonstrated that overt eye movements and foveal fixation are not correlated with the occurrence of apparent movement (Guilford \& Helson, 1929; Hulin \& Katz, 1934).

All subjects underwent a training period in which they saw drawings of a vertical and a horizontal line alternate at different duration rates, and in which they were instructed in the difference between the apparent motion of a single object and the appearance of two objects simultaneously. The simultaneity threshold for these lines was determined in six preliminary series. Each subject was then assigned to one of the six experimental conditions. In a 1-h session, six thresholds for each of the five randomized separations were determined. The first two threshold series served as practice series as well as a means for determining the threshold neighborhood for subsequent method-of-limits series. For these reasons, they were not included in the final data analysis. At the end of the experimental session, the observers were asked to describe generally the type of motion they had perceived while reporting "Yes."

\section{RESULTS}

Excluding the first two threshold series, an overall analysis of variance of thresholds on the remaining four series showed no main effect for ascending and descending series, and this factor did not interact with others. Therefore, the four thresholds were averaged for each observer to yield a single measure for each separation. An analysis of variance of these mean thresholds showed that all main effects and interactions were statistically significant $(p<.01$ in all cases).

\section{Types of Movement}

The types of movement described by subjects were grouped in the following categories.

(1) Third-dimensional movement: One square moves behind or in front of the picture plane; the square is rigid and approaches and recedes in depth from the observer, without changing in size.

(2) Plane movement: One square moves up and down in the picture plane. All subsidiary types of 
plane movement are listed as follows: (a) Beta movement-the square in motion does not change size. (b) Figural change-motion is accompanied by the square's changing in size, becoming small and then large; the square is plastic and not rigid. (c) Unidirectional movement-one square maintains its size and moves to the position of the second square; movement in the opposite direction does not occur.

(d) Fused movement-squares quite different in size fuse and move together, becoming one joint figure.

Third-dimensional movement was reported unanimously (five observers) in the presence of perspective context when the standard was paired with comparisons that decreased in size according to linear perspective. But, with the same comparison set, figural change was reported in the absence of perspective context. The only exception to unanimity of figural change was from one subject who reported unidirectional movement with the smallest comparison size. Figural change was also reported with reversed-perspective-sized squares, regardless of context. With the two smallest comparison sizes, however, a combination of figural change, unidirectional movement, and/or fused movement were reported by two subjects in each context condition. Finally, beta movement was unanimously reported when the standard and comparison were the same in objective size, regardless of context.

The thresholds for movement in various conditions were compared by pooling over separations. The effect of context type was significant for perspective size $[F(1,24) \geqslant 124.80, p<.01]$ and for identical size $[F(1,24) \geqslant 6.99, p<.05]$, but not for reversed-perspective size. Also, no difference was found between the mean thresholds for reversed- perspective and perspective size in the absence of perspective context. Furthermore, with regard to perspective context, the mean threshold for perspective size was greater than that for reversed-perspective size $[F(1,24) \geqslant 125.20, p<.01]$, and, in turn, the latter was greater than that for identical size $[F(1,24)$ $\geqslant 8.96, \mathrm{p}<.01]$.

The findings that the mean threshold for thirddimensional movement was greater than that for figural change and that, in turn, the mean threshold for figural change was greater than that for beta movement are in agreement with previously reported results (Kolers \& Pomerantz, 1971; Orlansky, 1940). Third-dimensional movement, however, occurred with perspective-size change only if the squares were congruent with the foreshortened depth gradient. Because the perceived size in apparent movement remained constant, it appears that retinal similarity is not necessary for apparent movement without size changes. This constancy of size in apparent motion suggests that the principle of figural similarity also depends on perceived size.

\section{Analysis of Separation}

Figure 2 presents the minimum field duration for each comparison set as a function of picture-plane separation for both context conditions. For all comparison sets, regardless of context, the thresholds systematically increase with separation. Although the divergence of the comparison-set functions was greater for perspective $[F(8,96) \geqslant 15.60, p<.01]$ than for frontal-plane context $[F(8,96) \geqslant 3.67, p<$ .01 ], the simple two-way separation $\times$ context interaction indicated that the functions were divergent only for the perspective sizes $[F(4,96) \geqslant 15.31, p<$
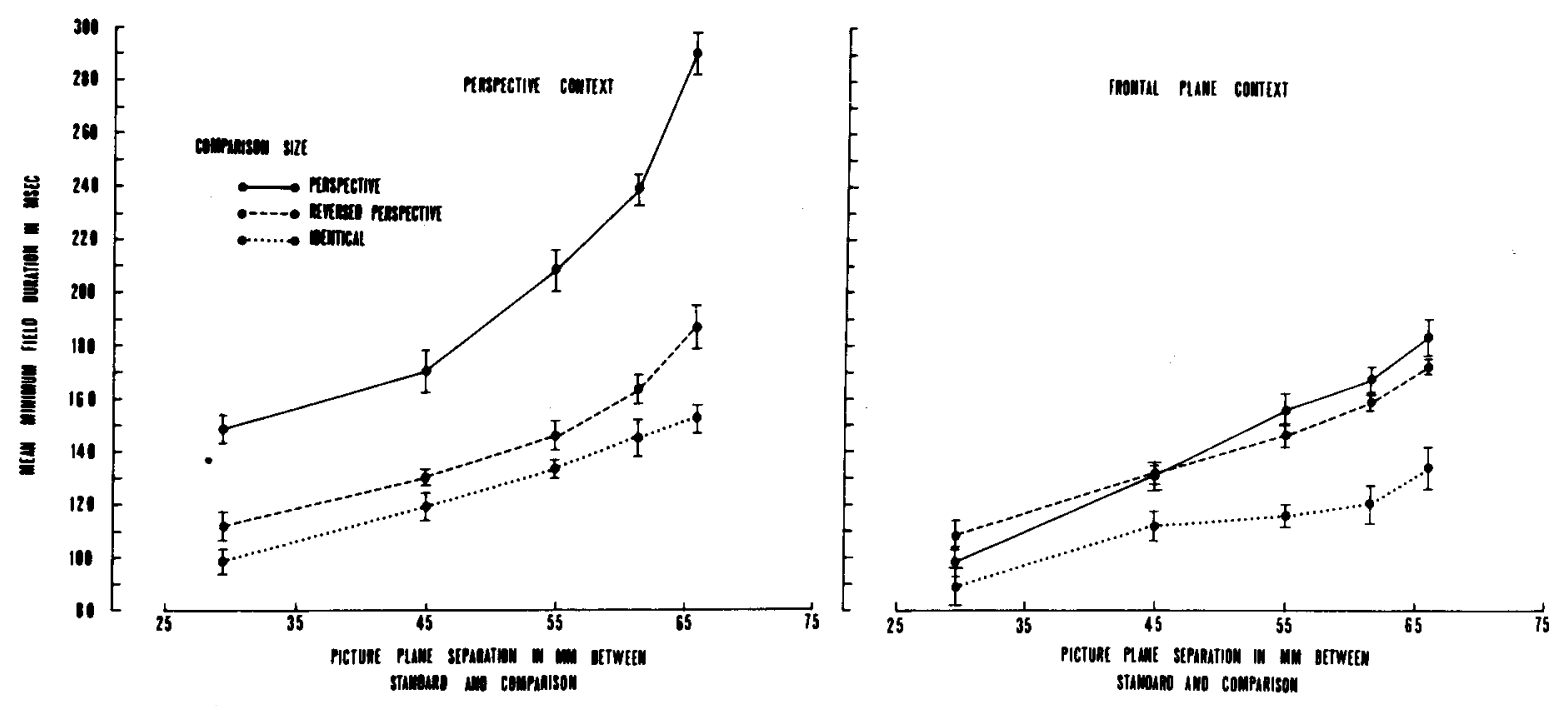

Figure 2. Mean minimum field duration (in milliseconds) required for apparent movement as a function of picture-plane separation (in millimeters) for each comparison set for both context conditions. Bars indicate \pm 1 standard error. 
$.01]$. Thus, the effects of perceived separation were not independent of comparison set.

Of greater interest, therefore, were trend analyses that either preserved picture-plane separation (unequally weighted coefficients) or employed hypothetical distal scale separations (equally weighted coefficients). For, if both perspective context and comparison set contributed to perceived separation, and if motion in a perspective array preserved textured gradient information, the thresholds should have increased linearly with distal scale, not with pictureplane separation. In order to simplify the presentation of these results, the linear trends are not discussed, since these trends were all statistically significant $(p<.01)$.

For the perspective comparison sizes, Figure 2 shows a nonlinear function for perspective context and a linear function for frontal-plane context. Only the perspective context condition showed significant quadratic $[\mathrm{F}(1,24) \geqslant 103.75, \mathrm{p}<.01]$, cubic $[\mathrm{F}(1,24)$ $\geqslant 6.04, p<.05]$, and quartic $[F(1,24) \geqslant 5.44, p<$ $.05]$ trends. The only other condition that showed a significant higher order trend (quadratic) was the reversed-perspective sizes with perspective context $[F(1,24) \geqslant 17.04, p<.01]$. The former higher order polynomial components unambiguously suggest that the thresholds increased at a rate greater than proximal separation and, hence, with a perceived extent in the third dimension greater than objective separation. Consequently, one would expect these same thresholds to increase linearly with the relative distal scale of pictorial depth. Therefore, the same data are presented in Figure 3 as a function of equally spaced separations. Unlike Figure 2, for the perspective sizes, Figure 3 shows an approximately linear function for perspective context and a curved downward function for the frontal-plane context. Both conditions showed significant quadratic trends, but in opposite directions [respectively, $F(1,24) \geqslant$ $12.99, \mathrm{p}<.01 ; \mathrm{F}(1,24) \geqslant 11.50, \mathrm{p}<.01]$. The only other condition that showed a significant higher order trend (cubic) was the identical-sized comparison set without perspective context $[F(1,24) \geqslant 5.66$, $\mathrm{p}<.05]$. The results make evident that apparent movement in the stimulus plane is directly related to separation in the stimulus plane, that is, that thresholds for figural change and beta movement increase linearly with picture-plane separation. But, on the other hand, thresholds for third-dimensional movement increase approximately linearly with pictorial representation of depth.

The discrepancies between experimental data (Figure 2) and expectations (Figure 3) that need attention are the functions for the reversed-perspective sizes. Why both increased linearly, although they had been expected to increase negatively, in Figure 3 is not immediately obvious. The same also holds for why the quadratic trend was significant with perspective context in Figure 2. But it should be noted that more than one type of movement was reported by some observers for pairs highly disparate in size, resulting in inflections of thresholds for apparent movement. In a more general sense, however, the violations could have been due to impoverished twodimensional representations of depth as well as to unrepresentative designs (Brunswik, 1956). Anomalies
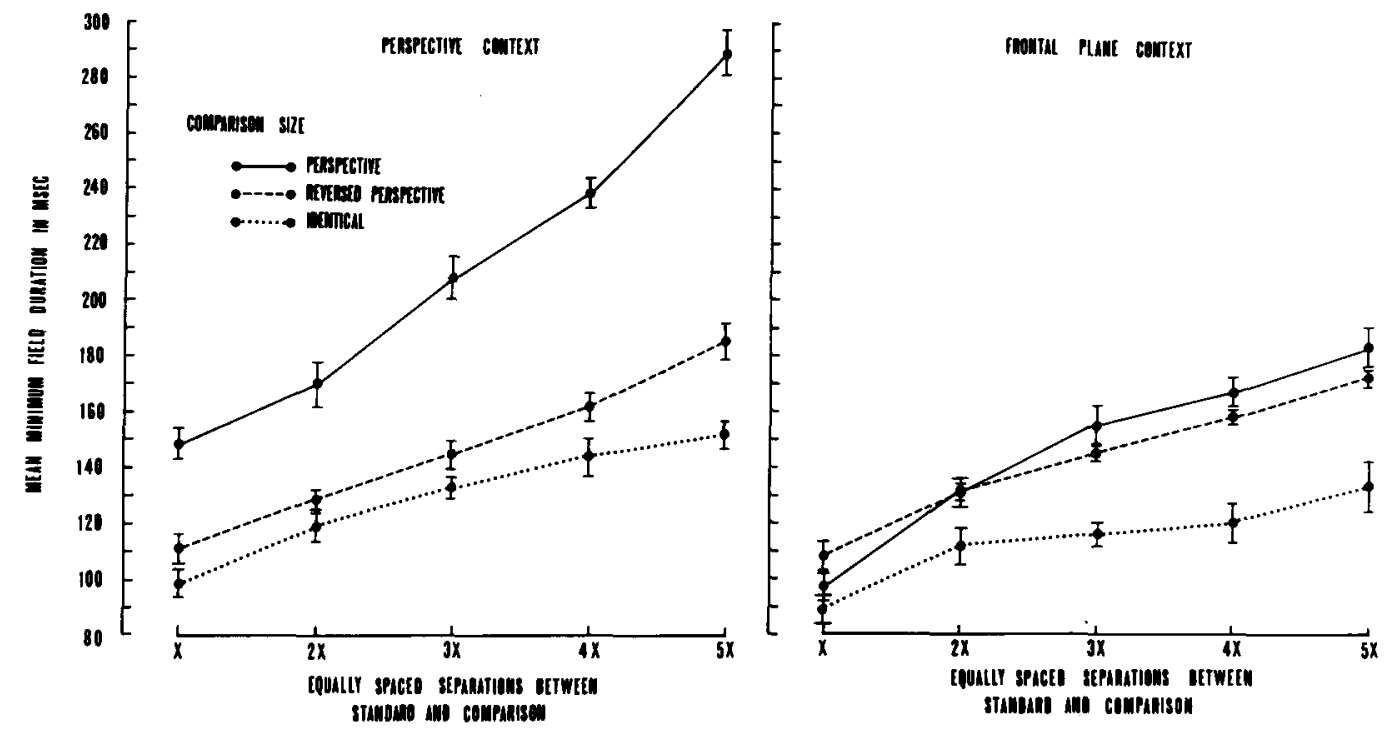

Figure 3. Mean minimum field duration (in milliseconds) required for apparent movement as a function of equally spaced separations for each comparison set for both context conditions. Bars indicate \pm 1 standard error. 
are not unprecedented whenever perceptual constancies are investigated under cue-reduction situations (Epstein, 1977).

\section{DISCUSSION}

The present study suggests that more time is needed to construct a rigid depth change in phenomenal space than to achieve either a change of size or simple plane movement because the path of the perceived movement traverses a greater distance than that perceived when two stimuli appear simultaneously in the picture plane (Figure 2). Even though phenomenal extent is quite an elusive variable to manipulate experimentally-as many experiments on the size-distance invariance hypothesis, for example, have demonstrated (see Epstein, 1977)-this extent was, nevertheless, approximated by rescaling stimulus separations as a function of hypothetical distal separation (Figure 3). The resultant function is consistent with the view that phenomenal extent is approximately isotropic because the function increased approximately linearly with equally spaced separations. This supports Corbin's thesis that apparent movement occurs at a postconstancy level of the perceptual system and, furthermore, that Korte's law is governed by the same variables that underlie perceptual constancies in pictorial arrays.

But why should this be so? We believe that Gibson's $(1950,1979)$ psychophysical approach to space perception provides a framework that can assimilate the obtained size constancy in apparent motion. According to Gibson, size constancy "is a by-product of the constant scale of the visual world at different distances. Scale, not size, is actually what remains constant in perception"' (1950, p. 181, italics ours). In two-dimensional pictorial arrays, size constancy occurs when proximal size is congruent with the representation of pictorial depth, because a constant distal size subtends a constant number of textural units. Since size is judged relative to some unit, in a pictorial array this unit is invariant. Hence, Korte's law depends on perceived depth-size separation and not on actual (retinal) size and separation. In our displays, it is the gradient of texture that provides a constant scale against which sizes are defined and evaluated.

Consistent with our interpretation that it is the properties of scale that matter for apparent movement in phenomenal space are interpretations from studies involving chronometric analysis of distal-size judgments in pictorial arrays (Pringle \& Uhlarik, 1982; Uhlarik et al., 1980). For instance, Pringle and Uhlarik (1982) found that reaction time for comparative ("same-different") judgments of distal sizes depended neither on absolute distance of stimulus size from the observer nor on proximal-size in- formation, but rather on the relative distance between sizes as defined by the distal scale implicit in pictorial arrays. The greater the relative distance ratio, the more time was required for "same" judgments. For the subject to have accomplished these comparative judgments, Pringle and Uhlarik (1982) suggested that, regardless of whether the stimulus itself or merely its scale transection was funneled through perceived depth, the properties of scale provided to the subject the "grammar" for preserving distal size throughout the array. In the context of apparent movement, however, we suggest that the stimulus transection of scale is funneled through depth, since stimulus funneling could have been possible in the reversed gradient-size correlation condition. But, instead, movement in depth was not reported and the thresholds did not increase nonlinearly with picture-plane separation. If such a possibility had occurred, the type of movement would have been an implosion and explosion of size in depth. An invariant size moving in phenomenal space, on the other hand, is consistent with the funneling of scale transection because proximal size, regardless of position in array, was defined by texturegradient scale.

Perhaps the neglect of texture-gradient scale accounted for the lack of constancy found by Attneave and Block (1973). We essentially replicated their results in showing differences by varying the type of context while keeping the size of the squares the same (Figure 2). But, while perspective context systematically increased the threshold, both functions increased linearly with picture-plane separation. Even if beta movement had not been reported, the linearity, in itself, would not have excluded the possibility that apparent movement varies with retinal separation, for the linearity suggests that the thresholds increased at a rate commensurate with that of picture-plane separation. That such a separation was the critical variable is further suggested by the apparent nonlinearity of the threshold functions shown in Figure 3, although only that for frontal-plane context was statistically significant.

It is not surprising, therefore, that Attneave and Block found less than perfect constancy, especially when the perceived separation threshold was considered against absolute (real) separation as a criterion for constancy. But rather than attributing this underconstancy to unnatural viewing conditions, we suggest that an invariant scale is not explicitly available throughout the array. In fact, contextual information for depth is in direct opposition to a scale unit of constant proximal size. This would also seem to be the case with reversed perspective size in a perspective array, in which figural change was usually reported and context type had no effect on thresholds. On the other hand, when gradient 
size is unambiguously defined, constancy is readily achieved by the "affordances" (cf. Gibson, 1979, pp. 127-143) of pictorial scale, not by an isotropic model that preserves distal events in an absolute fashion.

\section{REFERENCES}

Attnenve, F. Representation of physical space. In A. W. Melton \& E. Martin (Eds.), Coding processes in human memory. Washington, D.C: Winston, 1972.

Attnenve, F., \& Block, G. Apparent movement in tridimensional space. Perception \& Psychophysics, 1973, 13, 301-307.

Bnunswik, E. Perception and the representative design of psychological experiments. Berkeley: University of California Press, 1956.

Consin, H. H. The perception of grouping and apparent movement in visual depth. Archives of Psychology, 1942, 38, No. 273.

EPSTE IN, W. (Ed.). Stability and constancy in visual perception: Mechanisms and processes. New York: Wiley, 1977.

Grason, J. J. The perception of the visual world. Boston: Houghton Mifflin, 1950.

Gibson, J. J. The ecological approach to visual perception. Boston: Houghton Mifflin, 1979.

Gutlford, J. P., \& Helson, H. Eye-movements and the phiphenomenon. American Journal of Psychology, 1929, 41, 595-606.

Hulin, W. S., \& Katz, D. Eye-movements and the phi-phenomenon. American Journal of Psychology, 1934, 46, 332-334.

KofrKA, K. Principles of gestalt psychology. New York: Harcourt, Brace, 1935.

Kolers, P. A., \& Pomerantz, J. R. Figural change in ap- parent motion. Journal of Experimental Psychology, 1971, 87, 99-108.

OrLansky, J. The effects of similarity and differences in form on apparent visual movement. Archives of Psychology, 1940, 35, No. 246.

Pringle, R., \& Uhlarik, J. Comparative judgments of distal size: A chronometric analysis. Perception \& Psychophysics, 1982, 32, 178-186.

Sgro, F. J. Beta motion thresholds. Journal of Experimental Psychology, 1963, 66, 281-285.

Smith, O. W., \& Gruber, H. Perception of depth in photographs. Perceptual and Motor Skills, 1958, 8, 307-313.

Smith, O. W., Smith, T. C., \& Hubbard, D. Perceived distance as a function of the method of presenting perspective. American Journal of Psychology, 1958, 71, 662-675.

Uhlarik, J., Pringle, R., Jordan, K., \& Misceo, G. Size scaling in two-dimensional pictorial arrays. Perception \& Psychophysics, 1980, 27, 60-70.

WERTHEIMER, M. [Experimental studies on the seeing of motion.] In T. Shipley (Ed. and trans.), Classics in psychology. New York: Philosophic Library, 1961.

\section{NOTE}

1. While duration is kept constant, usually the onset-to-onset asynchrony has been used to measure apparent movement, for interval time decreases as duration time increases (Korte's fourth law). Our choice of keeping interval time constant while varying duration time was made in view of Sgro's (1963) results, which show that if duration time is above $50 \mathrm{msec}$, interval time approaches asymptote.

(Manuscript received December 7, 1981; revision accepted for publication December 2, 1982.) 